\title{
Time-Series Model for Wireless Fading Channels in Isotropic and Non-Isotropic Scattering Environments
}

\author{
P. Sharma, Member, IEEE
}

\begin{abstract}
An autoregressive (AR) model is presented for isotropic and non-isotropic scattering environments characterized by Rice factor $0 \leq K<\infty$. It is shown that at least a second order AR process is required to model the damped sinusoidal autocorrelation function (ACF) inherent in such environments. The model parameters are obtained by minimizing the squared error between the actual and the estimated ACF. This approach is shown to improve the ACF approximation as compared to the traditional Yule-Walker method. The model based simulator exhibits an order of magnitude gain in time complexity as compared to the sum of sinusoids method.
\end{abstract}

Index Terms-Time-series model, isotropic scattering channel, non-isotropic scattering channel, fading channel.

\section{Channel Features}

$\mathbf{C}$ ONSIDER a signal transmitted through a wireless channel modulated using a carrier with wavelength $\lambda_{c}$. Let index $m$ denote the number of multiple paths taken by the transmitted signal before arriving at the receiver. For an angle of arrival (AoA) $\theta_{m}$, the mobile velocity $v$, considered constant over the observation interval, causes a Doppler frequency shift $D_{m}$ of

$$
D_{m}=f \cos \theta_{m}
$$

where $f=\frac{v}{\lambda_{c}}$. The Doppler shift and the average received signal power $\Omega_{p}$ influence correlation properties of the received signal. The autocorrelation function (ACF) of inphase and quadrature components $x(t), y(t)$ of the received signal is given by [1]

$$
\rho_{x x}(\tau)=\rho_{y y}(\tau)=\frac{\Omega_{p}}{2} E\left[\cos \left(2 \pi D_{m} \tau\right)\right]
$$

and the cross-correlation $(\mathrm{CCF})$ function as

$$
\rho_{x y}(\tau)=-\rho_{y x}(\tau)=\frac{\Omega_{p}}{2} E\left[\sin \left(2 \pi D_{m} \tau\right)\right]
$$

Substituting $D_{m}$ from (1) in (2), (3) and taking the expectation $E[$.$] over the probability density function (PDF) of AoA f_{\Theta}(\theta)$ gives

$$
\begin{aligned}
& \rho_{x x}(\tau)=\frac{\Omega_{p}}{2} \int_{0}^{2 \pi} f_{\Theta}(\theta) \cos (\omega \tau \cos \theta) d \theta \\
& \rho_{x y}(\tau)=\frac{\Omega_{p}}{2} \int_{0}^{2 \pi} f_{\Theta}(\theta) \sin (\omega \tau \cos \theta) d \theta
\end{aligned}
$$

where $\omega=2 \pi f$. In an environment where scattered and specular components co-exist, the effect of $f_{\Theta}(\theta)$ and $\Omega_{p}$ can

Manuscript received May 26, 2004. The associate editor coordinating the review of this letter and approving it for publication was Dr. Zoran Zvonar.

The author is with the Center for Electronics Design and Technology (CEDT), Indian Center of Science, Bangalore, India (email: prsharma@att.net).

Digital Object Identifier 10.1109/LCOMM.2005.01022. be characterized in terms of the individual contributions of the line-of-sight (LoS) and the scattered signal.

The suburban macrocells are characterized by reflectors that are much smaller in dimension than the elevation of the basestation (BS). As a result, an unobstructed LoS signal exists between the BS and the mobile. Assuming LoS arrival at an angle $0 \leq \theta_{0} \leq 2 \pi$, the PDF of AoA $f_{\Theta_{l o s}}$ can be written as

$$
f_{\Theta_{\text {los }}}(\theta)=\left\{\begin{array}{lc}
1, & \theta=\theta_{0} \\
0 & \text { otherwise }
\end{array}\right.
$$

Local reflections at the receiver give rise to an isotropically scattered signal resulting in the PDF of AoA $f_{\Theta_{s}}^{(I)}(\theta)$ to be

$$
f_{\Theta_{s}}^{(I)}(\theta)=\frac{1}{2 \pi}, \quad 0 \leq \theta \leq 2 \pi
$$

Solving (4) and (5) by considering the individual contributions of LoS and scattered components, correlation functions for isotropic scattering can be obtained as

$$
\begin{aligned}
\rho_{y y}^{(I)}(\tau) & =\frac{\Omega_{p}}{2} \frac{K}{K+1} \cos \left(\omega \tau \cos \theta_{0}\right) \\
& +\frac{\Omega_{p}}{2} \frac{1}{K+1} J_{0}(\omega \tau) \\
\rho_{x y}^{(I)}(\tau) & =\frac{\Omega_{p}}{2} \frac{K}{K+1} \sin \left(\omega \tau \cos \theta_{0}\right)
\end{aligned}
$$

where $J_{0}(\omega \tau)=1 /(2 \pi) \int_{0}^{2 \pi} \cos (\omega \tau \cos \theta) d \theta$ is the Bessel function of first kind and zeroth order and $1 /(2 \pi) \int_{0}^{2 \pi} \sin (\omega \tau \cos \theta) d \theta=0$. The Rice factor $K=\frac{\Omega_{l_{o s}}}{\Omega_{s}}$ is the ratio between the average power of the LoS signal $\Omega_{\text {los }}$ and the scattered signal $\Omega_{s}$ such that, $\Omega_{p}=\Omega_{l o s}+\Omega_{s}$. In microcells, the scattering is non-isotropic and the scattered field is restricted within $[-\mathcal{A}: \mathcal{A}], \mathcal{A} \neq 0$ due to obstruction of the transmitted signal by buildings. Among many distribution functions available to characterize non-isotropic scattering [2] [3], the following PDF

$$
f_{\Theta_{s}}^{(N)}(\theta)= \begin{cases}\frac{\pi}{4|\mathcal{A}|} \cos \left(\frac{\pi}{2} \frac{\theta}{\mathcal{A}}\right), & |\theta| \leq|\mathcal{A}| \leq \frac{\pi}{2} \\ 0, & \text { otherwise }\end{cases}
$$

is chosen in this work due to its versatility in representing different urban locations by appropriately choosing $\mathcal{A}$ [1]. The $\operatorname{LoS}$ arrivals obey (6) with AoA restricted between $\theta_{0} \leq|\mathcal{A}| \leq$ $\pi / 2$. Solving (4) and (5) by separately considering $\operatorname{LoS}$ and scattered components, correlation functions for non-isotropic scattering can be obtained as

$$
\begin{gathered}
\rho_{y y}^{(N)}(\tau)=\frac{\Omega_{p}}{2} \frac{K}{K+1} \cos \left(\omega \tau \cos \theta_{0}\right) \\
+\frac{\Omega_{p}}{2} \frac{1}{K+1} \frac{\pi}{4|\mathcal{A}|} \int_{-\mathcal{A}}^{\mathcal{A}} \cos \left(\frac{\pi}{2} \frac{\theta}{\mathcal{A}}\right) \cos (\omega \tau \cos \theta) d \theta \\
\rho_{x y}^{(N)}(\tau)=\frac{\Omega_{p}}{2} \frac{K}{K+1} \sin \left(\omega \tau \cos \theta_{0}\right) \\
+\frac{\Omega_{p}}{2} \frac{1}{K+1} \frac{\pi}{4|\mathcal{A}|} \int_{-\mathcal{A}}^{\mathcal{A}} \cos \left(\frac{\pi}{2} \frac{\theta}{\mathcal{A}}\right) \sin (\omega \tau \cos \theta) d \theta
\end{gathered}
$$


By virtue of the central-limit theorem, when $m \rightarrow \infty, x$ : $N\left(\mu_{x}, \frac{\Omega_{s}}{2}\right)$ and $y: N\left(\mu_{y}, \frac{\Omega_{s}}{2}\right)[1]$, where $\mu_{x}=\sqrt{\frac{\Omega_{l o s}}{2}} \cos \theta_{0}$ and $\mu_{y}=\sqrt{\frac{\Omega_{l o s}}{2}} \sin \theta_{0}$. When the $\operatorname{CCF} \rho_{x y}^{(I)}(\tau)=0$, the resultant envelope $z(t)=\sqrt{x^{2}(t)+y^{2}(t)}$ is Ricean distributed for $K>0$ and Rayleigh distributed for $K=0$.

\section{Autoregressive Model}

Consider discrete samples of Gaussian distributed $x_{n}=$ $x(n L \Delta t)$ and $y_{n}=y(n L \Delta t)$. The value $L=1$ when the data is sampled at symbol interval $\Delta t$ and $L>1$ when the sampling interval exceeds $\Delta t$. Components $x_{n}$ and $y_{n}$ can be separately modeled as auto-regressive (AR) time-series [4]. The AR model of order $p^{(.)}$is given by [7]

$$
\begin{aligned}
\hat{x}_{n} & =\sum_{j=1}^{p^{(\hat{x})}} \Phi_{j}^{(x)} x_{n-j}+\Theta_{0}^{(x)}+e_{n}^{(x)} \\
\hat{y}_{n} & =\sum_{j=1}^{p^{(\hat{y})}} \Phi_{j}^{(y)} y_{n-j}+\Theta_{0}^{(y)}+e_{n}^{(y)}
\end{aligned}
$$

where $\Phi_{j}^{(.)}$is the $\operatorname{AR}$ coefficient, $\Theta_{0}^{(.)}$is a moving average term that is proportional to $\mu_{x}, \mu_{y}$ and $e_{n}^{(.)}$is additive white Gaussian noise distributed as $N\left(0, \sigma_{E}^{2(.)}\right)$. The superscript (.) is used to denote the parameter specific to the variable enclosed within the bracket. AR parameters in general, are a function of the ACF. Since $x_{n}$ and $y_{n}$ are governed by identical ACF, $p^{(x)}=p^{(y)}=p$ and $\Phi_{i}^{(x)}=\Phi_{i}^{(y)}=\Phi_{i}$ will be used. Since the variances of $x_{n}$ and $y_{n}$ are equal, the standard deviation $\sigma_{E}^{(y)}=\sigma_{E}^{(x)}=\sigma_{E}$.

To choose $p$, consider (8) and (11). The ACF $\rho_{y y}^{(I)}(\tau)$ is the summation of a cosine and the Bessel function. The ACF $\rho_{y y}^{(N)}(\tau)$ can be expressed in a similar form by approximating the integral term as [5]

$$
\begin{array}{r}
\int_{-\mathcal{A}}^{\mathcal{A}} f(\psi(\theta)) \cos (\mathcal{N} \theta) d \theta \leq \frac{1}{2} \int_{-\pi}^{\pi} f(\psi(\theta)) \cos (\mathcal{N} \theta) d \theta \\
\leq \pi f\left(\frac{\mathcal{N} \pi}{2}\right) J_{\mathcal{N}}(\omega \tau)
\end{array}
$$

where $\mathcal{N}=\frac{\pi}{2 \mathcal{A}}, \psi(\theta)=\omega \tau \cos \theta$ and the function $f($.$) can$ take values $\cos ($.$) or \sin ($.$) . Using (15), (12) can be expressed$ as a sine function. In a non-isotropic scattering environment $\mathcal{N}$ is real valued such that, $\mathcal{A} \rightarrow \frac{\pi}{2}$ yields $\mathcal{N} \rightarrow 1$, and $\mathcal{N} \rightarrow \infty$ as $\mathcal{A} \rightarrow 0$. For ease of analysis considering only the integer and positive valued $\mathcal{N}$, the Bessel function can be expanded as an infinite summation [6] as

$$
J_{\mathcal{N}}(q)=\left(\frac{q}{2}\right)^{\mathcal{N}} \sum_{k=0}^{\infty} \frac{\left(-\frac{q^{2}}{4}\right)^{k}}{k ! \Gamma(\mathcal{N}+k+1)}
$$

where, the Gamma function $\Gamma(\mathcal{N}+k+1)=(\mathcal{N}+k)$ ! and $q=\omega \tau$. Approximating (16) in the limit $q \rightarrow 0$ yields

$$
\begin{aligned}
\hat{J}_{\mathcal{N}}(q) & \approx \lim _{q \rightarrow 0} J_{\mathcal{N}}(q) \\
& =\left(\frac{q}{2}\right)^{\mathcal{N}} \frac{1}{\Gamma(\mathcal{N})}\left[1-\frac{\frac{q^{2}}{4}}{\mathcal{N}+1}+O\left(q^{4}\right)\right]
\end{aligned}
$$

where $O($.$) is the asymptotic upper bound of the approxi-$ mation error that is of the order of $q^{4}$. Thus, $\hat{J}_{\mathcal{N}}(q)$ can be approximated by a parabolic function having symmetry about $q=0$.

Next, consider the error $\Xi=\left|J_{\mathcal{N}}(q)-\hat{J}_{\mathcal{N}}(q)\right|$ for $0 \leq$ $\mathcal{N}<\infty$ for a small range of values of $q$ before the first zero crossing occurs. If $\mathcal{N}=0$ as is the case in an isotropic scattering environment, the first zero of $\hat{J}_{\mathcal{N}}(q)$ occurs at $q=2$ and that of $J_{\mathcal{N}}(q)$ at $q=2.404$. Hence, $\hat{J}_{0}(q)$ can be expected to match with $J_{0}(q)$ in the range $0 \leq q<2$. For large values of $\mathcal{N}$, the first zero crossing of $J_{\mathcal{N}}(q)$ and $\hat{J}_{\mathcal{N}}(q)$ occurs at $q=\mathcal{N}+1.85575 \mathcal{N}^{1 / 3}+O\left(\mathcal{N}^{-1 / 3}\right)[6]$ and $q=2 \sqrt{\mathcal{N}+1}$ respectively. Therefore, the error $\Xi$ increases with $\mathcal{N}$.

The first order approximation of the Bessel function in (17) can be matched by a cosine function

$$
\hat{J}_{\mathcal{N}}(q) \approx A \cos (Q)=A\left[1-\frac{Q^{2}}{2 !}+\frac{Q^{4}}{4 !}-\frac{Q^{6}}{6 !} \cdots\right]
$$

where $Q=\left|\frac{q}{2 \sqrt{(\mathcal{N}+1)}}\right|$ and $A=\left(\frac{q}{2}\right)^{\mathcal{N}} \frac{1}{\Gamma(\mathcal{N})}$. Using the relationships in (15)-(18), ACFs in (8) and (11) can be approximated as a summation of two cosines resulting in another cosine wave. To model such an ACF, a second order AR model (AR-2) can be chosen due to its capability in generating a cosine $\mathrm{ACF}$ of the form

$$
\nu_{y y}(k)=\frac{\mathcal{D}^{k} \sin (2 \pi \mathcal{F} k+\varphi)}{\sin \varphi}
$$

when model coefficients satisfy [7] $0<\Phi_{1}<2$ and $-1<$ $\Phi_{2}<0$. The frequency of oscillation $\mathcal{F}$, the phase $\varphi$ and the damping coefficient $\mathcal{D}$ of $\nu_{y y}(k)$ are related to AR coefficients $\Phi_{i}$ as [7]

$$
\begin{aligned}
\mathcal{D} & =\sqrt{-\Phi_{2}} \quad \cos (2 \pi \mathcal{F})=\frac{\left|\Phi_{1}\right|}{2 \sqrt{-\Phi_{2}}} \\
\varphi & =\tan ^{-1}\left[\frac{1+\mathcal{D}^{2}}{1-\mathcal{D}^{2}} \tan (2 \pi \mathcal{F})\right]
\end{aligned}
$$

Since, $\nu_{y y}(k)$ is a cosine function, note that the largest error between $\nu_{y y}(k)$ and $\rho_{y y}^{(.)}(\tau)=\rho_{y y}^{(.)}(k \Delta t)$ occurs when $K=0$. At $K=0$, the contribution of $J_{0}($.$) in (8) and the integral$ term in (11) that can be approximated by the Bessel function, is maximum. As $K \rightarrow \infty$, the influence of $\hat{J}_{\mathcal{N}}($.$) diminishes$ and $\rho_{y y}^{(.)}(k \Delta t)$ approaches a cosine, thereby reducing the error $\zeta(N)=\frac{1}{2} \sum_{k=0}^{N}\left|\rho_{y y}^{(.)}(k \Delta t)-\nu_{y y}(k)\right|^{2}$. Traditionally, parameters $\Phi_{i}$ are chosen using Yule-Walker (YW) approach [7] that minimizes the error $\left|y_{n}-\hat{y}_{n}\right|^{2}$. In this work, instead of computing $\Phi_{i}$, parameters $\mathcal{D}$ and $\mathcal{F}$ are computed to minimize $\zeta(N)$ by arbitrarily choosing a finite number of data-points $N$ to satisfy $\frac{d \zeta(N)}{d N} \approx 0$. For $K=0$, parameters obtained by the minimization of $\zeta(N)$ approximates $\rho_{y y}^{(I)}(k \Delta t)$ correctly until the third zero crossing as compared to the first zero-crossing in YW approach. To optimize $\zeta(N)$, the subroutine $D N 2 F$ from AT\&T port mathematical subroutine library package is used in this work.

The remaining model parameters are related to $\Phi_{i}$ as $\sigma_{E}^{2}=$ $\sigma_{y}^{2}\left(1-\Phi_{1} \nu_{y y}(1)-\Phi_{2} \nu_{y y}(2)\right)$ and $\Theta_{0}^{(y)}=\mu_{y}\left(1-\Phi_{1}-\Phi_{2}\right)$. When $K>0$ and $\mu_{x} \neq \mu_{y}$, AR-2 models for $x_{n}, y_{n}$ differ in parameter $\Theta_{0}^{(\cdot)}$. 


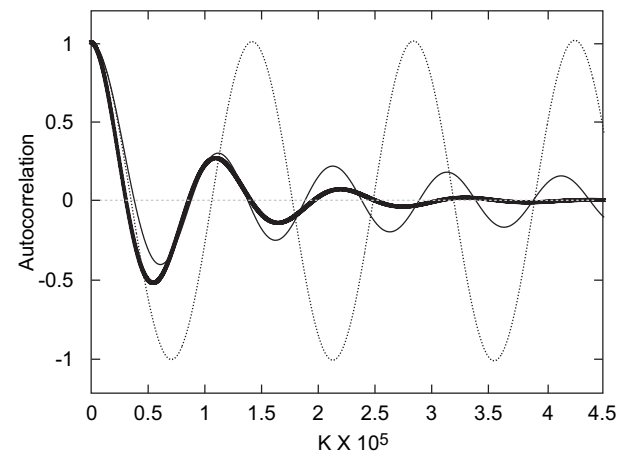

Fig. 1. $\mathrm{ACF}$ at $1 \mathrm{Mb} / \mathrm{s}, f=10 \mathrm{~Hz}$ : Solid line for $\rho_{y y}^{(I)}(\tau)$, thick solid line for $\nu_{y y}(k)$ obtained using minimization of $\zeta(N)$ and dotted line for $\nu_{y y}(k)$ obtained using YW method.

Next, it will be examined if CCF $\nu_{y x}(k)=E\left[\hat{y}_{n+k} \hat{x}_{n}\right]$ matches (9) and (12) when $x_{n}$ and $y_{n}$ are modeled by (13) and (14) respectively. Computing CCF from (13) and (14) gives

$$
\nu_{y x}(k)=\nu_{y x}(k-1) \frac{1-\Phi_{1}^{2}-\Phi_{2}^{2}}{\Phi_{1} \Phi_{2}}-\nu_{y x}(k-2)-C
$$

where,

$$
C=\frac{\Theta_{0}^{(x)} \Theta_{0}^{(y)}}{\Phi_{1} \Phi_{2}}+\frac{\Phi_{1}+\Phi_{2}}{\Phi_{1} \Phi_{2}}\left(\Theta_{0}^{(x)} \mu_{y}+\Theta_{0}^{(y)} \mu_{x}\right)
$$

is a constant. (22) can be represented as the following homogeneous difference equation

$$
\mathcal{B}^{2}-\mathcal{B} \frac{1-\Phi_{1}^{2}-\Phi_{2}^{2}}{\Phi_{1} \Phi_{2}}+1=0
$$

where the backward shift operator $\mathcal{B}$ is defined as $y_{k-1}=\mathcal{B} y_{k}$. $\mathrm{CCF} \nu_{y x}(k)$ is a cosine when the solution of (24) is complex [7]. A complex solution to (24) is obtained when the product of $1-\left(\Phi_{1}-\Phi_{2}\right)^{2}$ and $1-\left(\Phi_{1}+\Phi_{2}\right)^{2}$ is negative. AR-2 parameters obtained using the minimization of $\zeta(N)$ satisfy these conditions and yield a CCF that is a cosine function. To obtain CCF of the form of a sine function given by (9), (12), consider $\hat{x}_{n}$ and $\hat{y}_{n}$ with a shift of $\Delta$ to yield $\hat{\rho}_{y x}(k)=$ $E\left[\hat{y}_{n+k+\Delta} \hat{x}_{n}\right]$, where $\Delta=3 T / 4$ and $T$ is the period of ACF $\nu_{y y}(k)$ given by $1 / \mathcal{F}$.

\section{Performance}

The model performance is compared for $\rho_{y y}^{(I)}(k), K=0$ where the modeling error is the largest. A slow fading channel is considered in order to highlight the sensitivity of AR2 parameters to channel parameters. For a channel sampled at bit interval and supporting $1 \mathrm{Mb} / \mathrm{s}$ at Doppler frequency of $10 \mathrm{~Hz}$, the non-linear minimization of $\zeta(N)$ using $N=$ $450 \times 10^{3}$ points yields AR parameters $\mathcal{D}=0.999988005$ and $2 \pi \mathcal{F}=5.74322843 \times 10^{-5}$. The value of $N$ considers the correlation coefficients well beyond the third zero-crossing of the Bessel function. Increasing the Doppler frequency to $90 \mathrm{~Hz}$ and using $N=50 \times 10^{3}$ to maintain $f \tau N=4.5$ yields $\mathcal{D}=0.999892046$ and $2 \pi \mathcal{F}=5.16884486 \times 10^{-4}$. The error $\varepsilon_{\max }=\operatorname{Max}\left[\left|\rho_{y y}^{(I)}(k \Delta t)-\nu_{y y}(k)\right|^{2}\right]=0.071$ is recorded in both cases when $k \geq 0$ upper-bounded by the third zero crossing of the Bessel function is considered. The ACF $\nu_{y y}(k)$ obtained using aforementioned parameters for $f=10$

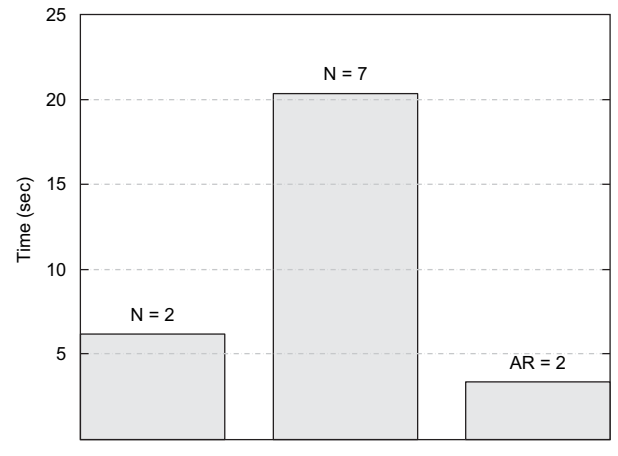

Fig. 2. Time complexity: SS and AR-2 model.

$\mathrm{Hz}$ is compared with $\rho_{y y}^{(I)}(k)$ in Fig. 1. The figure shows $\nu_{y y}(k)$ evaluated from (19) by substituting AR-2 parameters computed by (a) Minimization of $\zeta(N)(b)$ YW approach [7]. Approach $(a)$ slightly underestimates $\rho_{y y}^{(I)}(k)$ for small lags. Both the ACFs match until the third zero crossing after which $\nu_{y y}(k)$ decays to zero at a rate much faster than $\rho_{y y}^{(I)}(k)$. The ACF $\nu_{y y}(k)$ obtained using approach $(b)$ exhibits a slower damping and a phase offset as compared to $\rho_{y y}^{(I)}(k)$. The corresponding parameters yield a non-stationary AR process. For approach $(b)$, the error $\varepsilon_{\max }=1.15$ is two orders of magnitude larger than for approach $(a)$.

The time complexity of the simulation based on the AR-2 model is compared with the sum of sinusoids (SS) method [1] in Fig. 2. The real time (in seconds) required to generate $10^{6}$ samples is shown. The time is obtained using the LINUX csh command /usr/bin/time on a pentium III machine in a single-user environment. The time complexity of SS simulator is a function of the number of oscillators. The AR-2 model based simulator yields a two-fold gain for a minimum of two oscillators and a six-fold gain when oscillators in the SS simulator are increased to seven.

\section{CONCLUSIONS}

Though limited in representing the ACF of Rayleigh fading channel, the performance of AR-2 model improves as $K \rightarrow$ $\infty$ and desired ACF approaches a cosine. Choosing AR-2 parameters by minimizing the squared error between modeled and desired ACF improves the model accuracy in representing the ACF.

\section{REFERENCES}

[1] G. L. Stüber, Principles of Mobile Communication. Kluwer Academic Publisher, p. 44, 1996.

[2] A. Abdi et al., "Comparison of the level crossing rate and average fade duration of Rayleigh, Rice and Nakagami fading models with mobile channel data," 52nd Vehicular Technology Conference, 2000, vol. 4, p. 1850-1857, Sept. 2000.

[3] K. Anim-Appiah, "Complex envelope correlations for non-isotropic scattering," Electron. Lett., vol. 34, pp. 918-919, 1998.

[4] T. Eyceoz and A. Hallen, "Deterministic channel modeling and long range prediction of fast fading mobile radio channels," IEEE Commun. Lett., vol. 2, pp. 254-256, Sept. 1998.

[5] I. S. Gradshteyn, I. M. Ryzhik, and A. Jeffery, Table of Integrals, Series, and Products, (Fifth Edition). Academic Press, 1994, p.442.

[6] M. Abramowitz and I. A. Stegun, Handbook of Mathematical Functions with Formulas, Graphs and Mathematical Tables. National Bureau of Standards, 1964, p. 74, 360, 371.

[7] G. E. P. Box, G. M. Jenkins, and G. C. Reinsel, Time Series Analysis: Forecasting and Control. Prentice Hall, 1984, p. 56, 60-61, 120-125. 